6. That some cases, thought at the time to be cancer, too extensive for removal, may alter gastro-enterostomy clear up completely and get quite well

7. That in cases of disease of the cardiac end of the stomach extensive for removal, the operation of gastrostomy may considerably prolong life and prove of great comfort to the patient by preventing death from starvation.

8. That even where the disease is too extensive either for removal or for a gastro-enterostomy being performed with a fair chance of success, the operation of jejunostomy may occasionally prove of service to the patient.

9. That where a radical operation can be performed the thorough removal of the disease may bring about as much relief to the patient as does the operation for the removal of cancer in the breast, uterus, and other organs of the body and that in some cases a complete cure may follow.

\section{THE SURGICAL TREATMENT OF GASTRIC ULCER.}

B× C. MANSELL MOULLIN, M.D.Oxon., F.R.C.S., Senior Surgeon and Lecturer' on Surgery at the London Hospital ; Examiner in Surgery at the University of Cambridge.

Casms of gastric ulcer have hitherto been regarded as entirely the property of the physicians. Only when perforation or some similar accident has occurred is it admitted that the matter has passed out of their hands, and that a surgeon is required. Quite exceptionally, in a chronic case, when the patient has been brought to death's door by ceaseless pain and vomiting, when the power of repair has been reduced to a minimum by years of starvation, and it is evident that life under such conditions is no longer possible, and is hardly desirable, exceptionally, then, surgery is thought of, and a sargeon is asked to do, as a forlorn hope, what might have been done zears before with a splendid prospect of success. But even 80 much as this is a rare concession. Why this is so is, perhaps, not difficult to understand. One reason is the the want of acquaintance with, and as a result the want of trust in, the recent advances that have been made by surgery. Another, and the chief one I think, is the popular though certainly erroneous impression that the results of the present method of treatment are satisfactory. It seems to be the general opinion that the rate of mortality among those suffering from ulcer of the stomach is inconsiderable, and that relapses are unusual. The complications that follow, many of them of the gravest character, appear to be regarded a inevitable, to be met if they can be, but not to be prevented. In brief, what is called with, I am afraid, rather deceptive cynicism, the expectant method of treatment is apparently looked upon as doing all that can possibly be done.

Unhappily this optimistic view is very far from being correct. There is ample room for improvement, and I for my part look forward confidently to the time when chronic ulcer of the stomach will be regarded as an indication for operation, just as much as chronic inflammation of the appendix, and as early, if the object of the operation is to cure the patient; and when the complications that render acute ulceration so serious will be met at once in the same way, before it is too late, instead of allowing the patient to sink into an almost hopeless st ate, and then throwing the discredit of failure upon surgery.

I may at once disarm criticism by aring that $I$ have not the slightest intention of advocating operation as a general mode of treatment for all cases of ulcer of the stomach, or of rem sving this disease from the care of the physician. Ulcer of the stomach is and always will be more medical than surgical. I am perfectly content to leave the general treatment in the hands of physicians, and therefore I am not concerned in the least with the slighter cases of gastric ulcer, those for example which are treated as out-patients at hospital, and the majority-the great majority I should say-of th sse which you meet in private practice. They do not enter into the question. No one suggests, or has ever suggested, that they could possibly require an operation. So far as my present subject is concerned, I leave them upon one side entirely: I am going to deal only with the graver casesthnse which are sufficiently serious to be admitted into a hospital and tre ted as in-patients. This I suggest should be taken as the standard, and the question I wish to lay before you is simply whether, so far as these cases are concerned, the present method of treatment is really satisfactory, or whether the time has not come when an attempt should be made to obtain something better.

The results of the present methods of treatment can only be judged by collecting statistics. I know the fallacy of this. One distingnished surgeon, long since dead, once said to me that statistics could prove anything. His friends were kind enough to say that his remark was quite true of his own. So, instead of taking other people's, or working them out for myself, I asked Dr. Bulstrode, of the London Hospital, if he would collect them for me. This he very kindly did. $\mathrm{He}$ collected and analysed the statistics of all the cases admitted into the London Hospital for gastric ulcer from the beginning of 1897 down to August, 1902. They were just 500 in number, 98 men and 402 women. Ont of this number, no less than 48 (nearly 10 per cent:) died from peritonitis; 13 (or 2.5 per cent.) from haematemesis; and 28 (5.5 per cent.) from other causes. The total number of deaths was 89, or approximately 18 per cent., taking all the cases together. Had all the slighter ones been left out, and only those reckoned in which the gravity of the symptoms suggested the possibility of operation being advisable, the rate of mortality, bad as it is, would have been far higher.

The question of the actual rate of mortality, however, is not the only one that requires to be considered; 82 per cent. of the cases of gastric ulcer admitted into the London Hospital were discharged as relieved or cured. It would be exceedingly interesting to ascertain what was the relative proportion of these two classes; how many were really cured; that is to say, left the hospital perfectly well, without suffering pain or inconvenience when taking ordinary diet, and, what is especially important, remained well. Such a thing, unfortunately, is not possible. Some idea can, however, be formed by ascertaining the number of patients admitted into hospital with gastric ulcer who had already been treated for and cured of similar attacks. Dr. Bulstrode has done this for me, and has calculated that out of the 500 cases no less than 211 (42 per cent.) had suffered in this way previously. In 116 there had been only one previous attack; in $4^{1}$ there had been two; in 15 , three; and in 39 four or more. In other words, gastric ulcer recurs or relapses in at least two-filths of the cases which are apparently cured.

$B$ ad, however, as this sounds, it is not yet the worst. These figures do not include any of the cases admitted suffering from what may be called the late sequelae and complications of gastric ulcer. They do not take into account the cases admitted for pyloric stenosis (which Gerhardt and Warren both estimate at Io per cent.), hour-glass contraction, gastric dilatation, chronic dyspepsia, constant vomiting, perigastric adhesions, cardiac stenosis, and those numerous other disorders caused by the cicatrization of ulcers healed long since. Nor do they take into account those cases in which carcinoma has developed at the site of an old ulcer, which Hauser estimates at quite 6 per cent. When allowance has been made for all of these cases, which certainly cannot be described as cured, for the 18 per cent. of deaths. and the 42 per cent. of relapses, it must be admitted that the proportion of patients suffering from gastric ulcer of sufficient severity to require treatment in hospital, which is discharged as cured in the full sense of the term, in the sense in which a surgeon would use it after an operatiın, is not a very high one, not nearly 80 high as is generally imagined.

I do not think I shall be thought guilty of exaggeration, if I say that there is ample room for improvement here; and that if only the gravest of these cases had been selected from among the rest and treated by operation, not only would man more lives have been saved, but a vast amount of suffering would have been avoided.

My next point is to show that operation in these cases is not only feasible, but is attended by a sufficient measure of success to justify its recommendation. For this I shall rely entirely upon my own cases, and I shall confine myself to those in which I have operated directly because of the presonce of an active gastric ulcer. Operations performed for perforation, pyloric stenosis, perigastric abscesses, and the other complications and sequelae of gastric ulcer do not enter into the question. From my point of view they are too late. The gastric ulcer should have been operated upon before they occurred.

I have operated upon 15 cases in all, with 2 deaths. In 12 of these cuses the ulcer was excised or ligatured in various ways. In two gastro-jejunostomy was performed as the ulcer could not be dealt with locally; and in one the ulcer proved to be in the duodenum and not in the stomach. In four of these cases the immediate cause for operation was persistent 
pain and vomiting. These all recovered. In the other eleven the immediate cause was haematemesis, and in six of these the loss of blood had been so severe that transfusion had to be performed en the table or immediately after. Two of these died. They were both in extremis at the time of the operation, and as the notes have already been published I will not trouble you with the details of them.

I may add that I have been consulted with a view to operation in three other cases of recent haematemesis; but the condition of the patients was so hopeless that I would not undertaks the responsibility; and, as a matter of fact, they all died within twenty-four hours of my seeing them,

So much for the results of operation. Considering the desperate character of the majority of the cases in which it has been performed. I do not think that they compare badly with those that follow what is called expectant treatment. . No case died that had not already been brought literally to death's door. As the ulcer was removed entirely in all but two (in which its long continuance had rendered it impracticable) there can be no question of relapse; and for the same reason none of those complications that are caused by the persistent contraction at the base of a chronic sore can possibly occur. The question now arises when and under what circumstances operation can be advised.

With regard to this. I regret to say that I find it impossible to adopt a logical classification. The pathology of gastric alcer, which should furnish the foundation, requires revision. The appearance of a gastric ulcer during life is very different from what it is after death. I have several times found small bleeding fissures and abrasions with swollen overhanging. edges, and I have stopped the bleeding by ligaturing or excising them, but I have never yet seen in any operation the often described funnel-shaped gastric ulcer. I do not say that such an ulcer is never present during life, but I have never seen anything approaching it in appearance, and I cannot help thinking that I should have if it had been at all eommon.

Again, I can find no satisfactory pathological explanation for the development of what I have termed, fcr want of a better phrase, recurrent ulceration of the stomach. These cases stand by themselves. They are characterized by attacks of haematemesis, recurring again and again, after intervals of many months apparently of perfect health. I have operated upon several such cases during one of the attacks, and have never found anjthing but one or more bleeding fissures such as I have already described, undoubtedly of recent origin. There was no sign of a chronic ulcer. The bleeding had come entirely from. these fissures, which in these cases apparently form again and again. but I can find no explanation for their recurrence. So far, therefore, as the indications for operation are concerned, I must rely entirely upon the symptoms that happen to be present, and in speaking of these I think it will be more satisfactory to deal with acute and chronic ulceration separately.

In acute ulceration there are only two of these indicationsthreatened perforation and haemorrhage-and of these the former can rarely be diagnosed beforehand. The wall of the stumach itself is entirely devoid of sensation. It may be cut, or pinched, or even cauterized without the patient being aware of it; and accordingly perforation may occur suddenly from one moment to another, and has been known to do so without the patient having had the slightest warning. Such cases, of course, cannot be foreseen. They are quite beyond our power. But I do not think this is so with all. It is sometimes possible to say that an ulcer is spreading instead of healing; and though it is not possible to say whether it is spreading in breadth or depth, or both, the fact that it is spreading causes thought. The symptom to which I refer is the presence of persistent cutaneous hyperaesthesia in Head's epigastric triangle, especially when it is combined with a similar condition at the corresponding dorsal point. This means that there is something going on in the wall of the stomach, sufficiently grave to render the corresponding spinal nerve segment unduly sensitive. It has the same signification as cataneous tenderness over the side of the abdomen in inflammation of the appendix. It is, in short, an indication that the corresponding splanchnic nerve-in this case the one distributed to the wall of the stomach-is exposed to some source of irritation; and though cutaneous tenderness by itself is not sufficient to diagnose the presence of an ulcer, I am disposed to think that when the existence of an ulcer is shown by other evidence, it is a sufficient indication that the ulcer is not healing; and I should not hesitate to recommend exploration when this cutaneous hyperaesthesia is definite and well marked, supported by other signs and persists in spite of a thorough course of ordinary treatment.

The other indication for operation is more obvious. No one, I think, will refuse to admit nowadays that if haemorrhage from the stomach is so profuse as to place life in danger, an attempt must be made to expose the bleeding point. No patient should be allowed to bleed to death if there is a chance of stopping the haemorrhage. But there is a reason which renders this question not quite so simple as it appears to be. There is a peculiarity about haemorrhages from what are called acute ulcers of the stomach. They often persist, in spite of all that is done to check them, until the patient is apparently on the point of bleeding to death, and then stop of themselves. Hence there is always a risk that a serious operation may be performed unnecessarily, especially as the actual rate of mortality from this cause in cases of acute ulceration is not more than 3 per cent. My own impression is that each case must be judged upon its merits. I have seldom had the opportunity of advising delay, simply because a surgeon is seldom called in until the physician has exhausted every means at his command; but, as I have mentioned already, in 3 cases in which $I$ was called in I had to decline, it was too late; and in 6 in which I did operate transfusion was absolutely necessary. Four of these recovered.

The guide that I have always followed is the condition of the patient. A single haemorrhage is rarely fatal. Nearly always, if the stomach is given absoluterest, the bleeding will stop of itself. But if the pulge-rate continues to grow quicker and quicker, and particularly if the well.known restlessness and yawning of haemorrhage set in, I do not hesitate to recommend exploration. There is too great a probability that the haemorrhage is going on still, and that the blood is collecting in the bowel or the stomach. This was actually the case in one of my cases.

I believe the same plan should be followed if a second severe haemorrhage occurs within a few days of the first. This means that some vessel is bleeding. The chances are thast it is an artery, and that there is a hole in one side of it. The bleeding has stopped once. The clot that plugged the opening hns been removed by the action of the gastric juice, or the irritation which prevents the ulcer from healing has caused a further destruction of the wall, and the bleeding has returned. It is not wise to run the lisk of allowing this to happen a third time.

And I believe the same plan should be followed in cases in which the haemorrhage, though comparatively slight, returng again and again. Frequently repeated haemorrhages, it is well known, are apt to induce a condition of anaemia, which renders patients much more susceptible to other disorders, especially to tuberculous diseage. It has been estimated that upwards of 20 per cent. of those who suffer from this form of haematemesis, die subsequently from tubercle. If this is really true, and there appears to be very little doubt about it, this condition cannot be regarded as otherwise than very serious; and it is an open question whether an operation should not be performed at the first favourable opportunity, and the bleeding point secured, even though there may be only a 3 per cent. chance that the haemorrhage will prove directly fatal. Personally I think there is no doubt that this is the course that should be followed.

In chronic ulcer of the stomach indications for operation are much more simple. I hold that every case of chronic ulcer of the stomach, because it has become chronic, should be operated upon without further delay. The mere fact of its having become chronic is proof that the previous treatment has falled to cure it; and if one thorough trial of orjinary measures has been made, and has not succeeded, it is better that an operation should be performed without waiting further, than that the old measures, already discredited by failure, should be tried over and over again, each time in less fuvourable circumstances, until at last a condition is reached that is little short of hopeless.

Chronic ulcer of the stomach, an ulcer that has become chronic under, and in spite of, treatment, is practically besond cure, in the full sense of the term, except by operation. The patient may get relief for a time, but nothing more. The longer such an ulcer lasts the worse it becomes, and the greater the liability to complications which, if not fatal of themselves, only too often succeed in rendering life a burden. Perforation and general septic peritonitis, subphrenic and perigastric abscesses, ulceration extending into the liver or pancreas, perigastric adhesions, hour-glass contraction of the stomach, pyloric stenosis (in a very large proportion of cases), haemormorrhage from ulceration of the walls of the coronary or 
splenic arteries, or even of the aorta, and cancer in many instances, are simply the result of leaving things alone, of what is called expectant treatment; of allowing, in other words, the ulcer steadily to grow larger and the patient grow weaker. What proportion of cases die from these complications is difficult to estimate, but it is not a small one. Haemorrhage alone, as Dr. Wall has shown at the London Hospital is responsible for the deaths of 6 per cent. of the women, and $12 \frac{1}{2}$ per cent. of the men over 30 years of age admitted into the wards for haematemesis from ulcer of the stomach. The essential point is that hardly one escapes. It is no exaggeration to say that chronic ulcer of the stomach always leads to some complication or other, whether it continues to spread or whether it heals and cicatrizes; and that even if these complications do not prove directly fatal, they only too often succeed in rendering life miserable.

On the other hand, excision of such an ulcer, so long as it is confined to the mucous membrane and does nct greatly involve the sero-muscular coat, is comparatively simple. I have operated upon five such, three by excision and two by ligature of the whole thickness of the wall, and in each repair was rapid and complete. There is no reason why the operation should be followed by a higher rate of mortality than that which follows interval operations for inflammation of the appendix. Later, when the strength of the patient is broken down by years of chronic starvation, and all sorts of complications are present, the same operation may easily become one of the most difficult in surgers, so that not infrequently it has had to be abandoned. But this is not an argument against operation, but against delay.

The details of the operation and the mode of dealing with the ulcer I need not go into now as I have already fully described them elsewhere. The only thing I wish to say is that I am strongly in favour, wherever it is possible, of dealing with the ulcer itself, whether this is done by excision or by ligature.

Gastro-enterostomy should be reserved for cases in which this is impracticable, in which, for instance, the ulceration is multiple, or in which it obstinately recurs, or in which there is already some degree of stenosis at the pylorus or in the walls of the stomach. It places the stomach at rest, it is true, and so often enables the ulcer to heal; but it cannot stop bleeding (there are at least two cases on record in which the haemorrhage has continued and proved fatal, and I have been told of a third), and it is at best only a way of evading difficultits which are better met directly, before they have attained dimensions which render this impossible.

\section{CASE OF PARTIAL GASTRECTOMY, WITH REMARKS UPON THE TREATMENT OF MALIGNANT DISEASE OF THE STOMACH.}

By B. G. A. MOYNIHAN, M.S.LoND., F.R.C.S., Senior Assistant Surgeon, Leeds General Infirmary; Consulting Surgeon to the Skipton Hospital, and to the Mirfleld Memorial Hospital.

In a paper read before the Clinical Society on November 8th I 901 , I described an operation that seemed to me to fulfil certain requirements in the radical treatment of cancer of the pyloric portion of the stomach. By means of this operation I hoped that it would be possible to remove-

(a) The primary growth, and all the area likely to be involved in its increase.

(b) The lymphatic vessels draining the area in which the primary growth occurred.

(c) The lymphatic glands into which these vessels drained

The need for some more scientific method of dealing with cancer at or near the pylorus was obvious when the records of published cases were considered. In these the perform ance of "pylorectomy"-removal, that is, of the pylorus, and a margin of healthy stomach around the growth-had resulted in the experience that in the great majority of cases the patients eventually died of a local recurrence-a recurrence, that is, in the walls of the stomach. In order to discover the lines which an operation should follow if local recurrence were to be avoided, it was necessary to inquire into, first, the mode of invasion of the stomach by the growth the lines of spreading of the cancer from the original point affected, and the rapidity with which growth occurred along these lines; and secondly, the anatomy of the lymphatic syetem of the stomach, the direction in which the lymphatic vessels ran. and the position of the glands in which they terminated. The result of the investigations, so far as the first point was concerned, resulted in the following conclusions :

1. That malignant disease of the stomach begins in the majority of instances near the pylorus just below the lesser curvature.

2. That from this point it spreads most rapidly and most

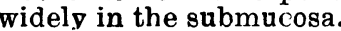

3. That the rate of growth towards the cardiac orifice is rapid, towards the duodenal side extremely slow. The duodenum is rarely affected extensively.

4. That the tendency of the growth is to drift towards the curvatures.

So far as the second point is concerned it was found that the lymphatic system of the stomach was eomparatively simple. There are three chief lymphatic areas of the stomach (Fig. I).

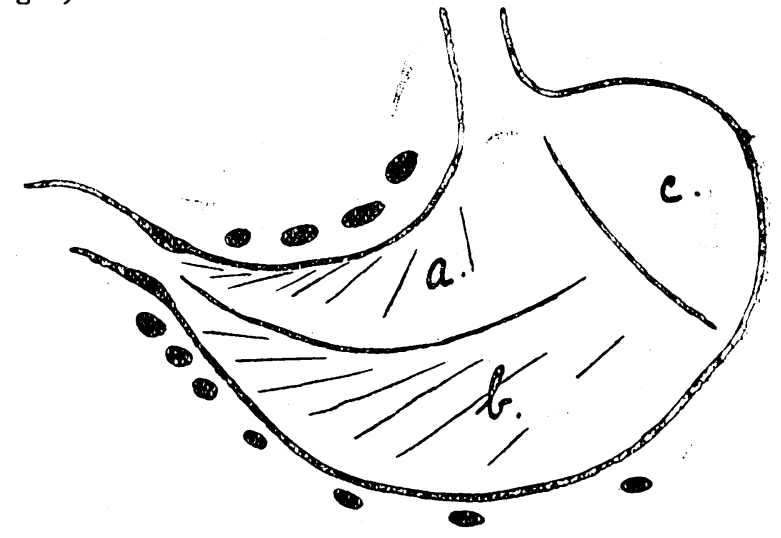

Fig. I. - The lymphatic vessels and glands of the stomach. C is the "isolated area."

In area along the lesser curvature from which the lymphatic vessels pass upwards and to the left into the coronary glands. The coronary glands lie along the artery of the same name. At the coeliac axis they become continuous with the glands along the upper border of the pancreas.

2. An area along the greater curvature from which the lymphatic vessels pass downwards and to the right into the glands lying along the greater curvature. These glands are more numerous near the pylorus, and from here pass to the head of the pancreas and become continuous with the hepatic group of glands which lie along the hepatic artery, and in part along the pyloric artery.

3. In addition to these two areas is a third, for which I suggested the name "isolated area." This area comprises the greater tuberosity of the stomach, the lower end of the oesophagus, and an area along the greater curvature as far, approximately, as the limit of supply of the left gastro-epiploic artery. Its lymphatic vessels pass downwards to the hilum of the spleen. The term "isolated area" seems singularly appropriate for this region, for it is very rarely affected by growth spreading upwards from the pylorus. An examination of a large number of specimens of pyloric cancer will show that this area remains unaffected till the last, and that its lymphatic vessels and glands are very rarely involved in the spread of the disease. An operation therefore which aims at eradicating the primary growth, the lymphatic vessels draining the area in which the primary growth occurs, and the lymphatic glands into which these vessels drain will involve a removal of all the stomach except the "isolated area," and of the glands along the lesser and greater curvatures. On the duodenal side, the first portion of the duodenum will the duodenal side, meved beyond this it will very rarely, if ever, be necessary to go. The following are the notes of the first case upon which I was able to carry out this operation. As the clinical history of the case is now complete it is desirable to publish the record:

W. H. S male, aged 3 . Three years ago the patient began to have pain after food, belching, and occasional vomiting. These troubles gradually and progressively increased. During the last six or seven months he has been losing weight; during the last two months the loss has been rapid. In his present state he is quite unable to take any solid food, and can in link liquids only in small quantity. The pain is not acute at any time now but here is a sense of constant heaviness and burning in the upper abdomen. When keeping erect, standing or sitting, he does not vomit, but within a few minutes of lying down a large quantity of brownish- 